\title{
A Random Multifractal Tilling
}

\author{
M. G. Pereira, G. Corso†, L. S. Lucena, and J. E. Freitas \\ International Center for Complex Systems and \\ Departamento de Física Teórica e Experimental, \\ Universidade Federal do Rio Grande do Norte, \\ Campus Universitário 59078 970, Natal, RN, Brazil. and \\ $\dagger$ Departamento de Biofísica, Centro de Biociências, \\ Universidade Federal do Rio Grande do Norte, \\ Campus Universitário 59072 970, Natal, RN, Brazil.
}

(Dated: December 7, 2018)

\begin{abstract}
We develop a multifractal random tilling that fills the square. The multifractal is formed by an arrangement of rectangular blocks of different sizes, areas and number of neighbors. The overall feature of the tilling is an heterogeneous and anisotropic random self-affine object. The multifractal is constructed by an algorithm that makes successive sections of the square. At each $n$-step there is a random choice of a parameter $\rho_{i}$ related to the section ratio. For the case of random choice between $\rho_{1}$ and $\rho_{2}$ we find analytically the full spectrum of fractal dimensions.
\end{abstract}

Keywords: multifractal, fractal spectrum, random object, complex systems 


\section{I - INTRODUCTION}

In the reference 1, 2] a deterministic multifractal tilling is developed to study percolation in multifractal systems. This object is a natural generalization of the square lattice in which it is possible to estimate analytically the full spectrum of fractal dimensions. Besides it exhibits a rich distribution of area among the blocks and a non-trivial topology, the number of neighbors varies along the object. The multifractal, however, is deterministic in its construction. In spite of its properties it lacks the random ingredients found in natural systems. In order to make the model more realistic we develop a random version of this model.

In this work we develop a multifractal that has the following characteristics. It has an infinite number of subsets each one with a distinct fractal dimension. For a specific case we determine analytically the dimension of each subset. The sum of all the subsets fills completely the square. The algorithm of construction of the multifractal has two or more free parameters, $\rho_{i}$, that are related to the stretching of its subsets. During the construction of the multifractal there is random choice of $\rho_{i}$ which makes the object non deterministic. For the special case of just one parameter $\rho=1$ the object degenerates into the square lattice. The extension of the model for an arbitrary dimension is trivial. Finally the algorithm for construction of the multifractal is simple and easily implemented in the computer.

Geologic structures can show multifractal characteristics [3, 4, 5, 6, 7] in physical properties like porosity, permeability, or sound velocity. In addition the rocks and strata are not deterministic objects, but present random features. These geologic systems that combine multifractality and randomness are natural candidates to be modeled by the proposed model.

The deterministic multifractal is an intuitive generalization of the square lattice. Suppose that in the construction of the square lattice we use the following algorithm: take a square of size 1 and cut it symmetrically with vertical and horizontal lines. Repeat this process $l$-times; at the $l^{\text {th }}$ step we have a regular lattice with $2^{l} \times 2^{l}$ cells. The setup algorithm of the deterministic multifractal is quite similar, the main difference is that we do 
not cut the square in a symmetric way, but according to a given constant ratio. The random multifractal is an extension of the deterministic model. In the random multifractal we use a non constant ratio in the algorithm setup.

The main objective of this work is to present a random multifractal tilling and determine its set of fractal dimensions for a specific case. The manuscript is organized as follows: in section 2 we present the deterministic multifractal and review the derivation of its set of fractal dimensions. In section 3 we develop the random multifractal and indicate a formula that estimate its set of fractal dimensions for the case of random choice between two given distinct $\rho_{i}$. Finally in section 4 we summarize the main differences between deterministic and random multifractal tillings and give our final remarks.

\section{II - THE DETERMINISTIC MULTIFRACTAL}

We start with a square of linear size 1 and a parameter $0<\rho=\frac{s}{r}<1$, for integers $r$ and $s$. The first step, $n=1$, consists of making one vertical section of the square. The square is cut in two pieces of areas $\frac{s}{r+s}=\frac{\rho}{1+\rho}$ and $\frac{r}{s+r}=\frac{1}{1+\rho}$ by a vertical segment. This process is shown in figure 1 (a), where we use $\rho=\frac{2}{3}$. The step $n=2$ consists of a horizontal cut with the same section $\rho$, this step is shown in figure 1 (b). At this point

there are four rectangular blocks: the largest one of area $\left(\frac{1}{1+\rho}\right)^{2}$, two of area $\frac{\rho}{(1+\rho)^{2}}$ and the smallest one of area $\left(\frac{\rho}{1+\rho}\right)^{2}$. We call a set of all elements with the same area as a $k$-set. At the step $n=2$ there are three $k$-sets.

In the third step, $n=3$, we repeat the same process of vertical section as in step $n=1$. At $n=4$ we make new horizontal sections. All the sections have the same ratio $\rho$. At the $n^{\text {th }}$-step there are $2^{n}$ blocks. The partition process produces a set of blocks with a variety of areas. At the step $n$ we have $n+1 k$-sets. The construction process of the deterministic multifractal is done by vertical and horizontal sections of the square. As the section ratio is always the same and the segment section is displaced in space the object shows self-affinity.

At the $n^{\text {th }}$-step of the algorithm, the partition of the area of the square follows the 
binomial rule:

$$
1=\sum_{k=0}^{n} C_{k}^{n}\left(\frac{s}{s+r}\right)^{k}\left(\frac{r}{s+r}\right)^{n-k}=\left(\frac{r+s}{r+s}\right)^{n}
$$

or:

$$
1=\sum_{k=0}^{n} C_{k}^{n}\left(\frac{\rho}{1+\rho}\right)^{k}\left(\frac{1}{1+\rho}\right)^{n-k}=\left(\frac{1+\rho}{1+\rho}\right)^{n} .
$$

The number of elements of each $k$-set in this case is $C_{k}^{n}$.

Each $k$-set determines a specific dimension. For the estimation of the spectrum of fractal dimension $D_{k}$ of an object $X$ we use the box counting method [8]. The object $X$ is immersed in the plane of real numbers $\Re^{2}$, we use the trivial metric. We cover the object by just-touching square boxes of side length $\epsilon$. Let $N(X)$ denote the number of square cells of side length $\epsilon$ which intersect $X$. If

$$
D_{X}=\lim _{\epsilon \rightarrow 0} \frac{\log N(X)}{\log \frac{1}{\epsilon}}
$$

is finite, then $D_{X}$ is the dimension of the $X$.

In our case the object $X$ is a $k$-set. Remember that the $k$-set corresponds to a set of rectangles of the same area. If the square is partitioned $n$ times we have $\epsilon=1 /(s+r)^{n}$. For each $k$-set the area of blocks (using $\epsilon$ area units) is done by:

$$
N_{k}=C_{n}^{k} s^{k} r^{(n-k)}
$$

where $C_{n}^{k}$ is the binomial coefficient that express the number of elements $k$-type, and $s^{k} r^{(n-k)}$ is the area of each element of this set. We put together all these elements to have the fractal dimension of each $k$-set:

$$
D_{k}=\lim _{\epsilon \rightarrow 0} \frac{\log N_{k}}{\log \frac{1}{\epsilon}}=\lim _{n \rightarrow \infty} \frac{\log C_{n}^{k} s^{k} r^{(n-k)}}{\log (s+r)^{n}} .
$$

The case $r=s=1$ is degenerated. In this situation the subsets of the lattice are composed uniquely by square cells of the same area. Therefore the tilling is formed by a single subset of dimension 2 .

Figure 2 shows the spectrum of $D_{k}$ for several values of $\rho$. We plot in the $x$-axis the normalized $k$-set, $k / n$, and in the $y$-axis the respective $D_{k}$. The dashed line corresponds to a $\rho$ that is very closed to 1 in this situation the blocks have almost the same area and $D_{k}$ is a symmetric curve. In the opposite situation $\rho \rightarrow 0$ we have curves that correspond 
to a tilling in which the blocks are very stretched in one direction. In the figure it is also depicted the curves corresponding to $\rho=0.4,0.6$ and 0.8 .

\section{III - THE RANDOM MULTIFRACTAL}

In the last section we use a constant $\rho$ through all the $n$-steps of the construction of the multifractal. A more realistic generalization of the above process is to use different values of the ratio $\rho$ along the growing process of the multifractal. In the general case a random $\rho_{i}$ is used at each step of the algorithm. In this work we explore in more detail the case where at each $n$-step of the construction of the multifractal we randomly choose between two rational values $\rho_{1}=s_{1} / r_{1}$ and $\rho_{2}=s_{2} / r_{2}$ for $r_{1}+s_{1}=r_{2}+s_{2}$. The picture of this process is similar to figure 1, but this time the section of the square does not follow a constant ratio making the partition of the square asymmetric.

We have in the algorithm of the random multifractal the following choices. At $n=1$ we divide the square in two blocks. There are two partition possibilities for the square: $\rho_{1} /\left(1+\rho_{1}\right)$ and $\left(1-\rho_{1}\right) /\left(1+\rho_{1}\right)$ or $\rho_{2} /\left(1+\rho_{2}\right)$ e $\left(1-\rho_{2}\right) /\left(1+\rho_{2}\right)$. At the second step, $n=2$, there are two possibilities for each former configuration. These are the three partition possibilities at step $n=2$ :

$$
\begin{aligned}
& \quad \rho_{1}^{2} /\left(1+\rho_{1}\right)^{2}, \rho_{1}\left(1-\rho_{1}\right) /\left(1+\rho_{1}\right)^{2},\left(1-\rho_{1}\right)^{2} /\left(1+\rho_{1}\right)^{2}, \rho_{1}\left(1-\rho_{1}\right) /\left(1+\rho_{1}\right)^{2}, \\
& \quad \text { or } \rho_{1} \rho_{2} /\left(1+\rho_{1}\right)\left(1+\rho_{2}\right),\left(1-\rho_{1}\right) \rho_{2} /\left(1+\rho_{1}\right)\left(1+\rho_{2}\right), \rho_{1}\left(1-\rho_{2}\right) /\left(1+\rho_{1}\right)\left(1+\rho_{2}\right), \\
& \left(1-\rho_{1}\right)\left(1-\rho_{2}\right) /\left(1+\rho_{1}\right)\left(1+\rho_{2}\right), \\
& \quad \text { or } \rho_{2}^{2} /\left(1+\rho_{2}\right)^{2}, \rho_{2}\left(1-\rho_{2}\right) /\left(1+\rho_{2}\right)^{2},\left(1-\rho_{2}\right)^{2} /\left(1+\rho_{2}\right)^{2}, \rho_{2}\left(1-\rho_{2}\right) /\left(1+\rho_{2}\right)^{2} .
\end{aligned}
$$

Note that there are not four configurations but only three. In fact, it is the same to make a section $\rho_{1}$ after $\rho_{2}$, or $\rho_{2}$ after $\rho_{1}$.

At the step $n, \rho_{1}$ is chosen $l$ times and $\rho_{2}$ is chosen $m$ times for $l+m=n$. At each step the areas of the blocks are obtained by the multiplication of the area of the block of the former step. Therefore the partition of the area of the square is done by the following equation:

$$
1=\sum_{i=0}^{l} \sum_{j=0}^{m} C_{i}^{l}\left(\frac{\rho_{1}}{1+\rho_{1}}\right)^{i}\left(\frac{1-\rho_{1}}{1+\rho_{1}}\right)^{l-i} C_{j}^{m}\left(\frac{\rho_{2}}{1+\rho_{2}}\right)^{j}\left(\frac{1-\rho_{2}}{1+\rho_{2}}\right)^{m-j}
$$




$$
=\left(\frac{1+\rho_{1}}{1+\rho_{1}}\right)^{l}\left(\frac{1+\rho_{2}}{1+\rho_{1}}\right)^{m} .
$$

This formula is analogous to equation (2) of the deterministic case. The occurrence of blocks of the same $k$-sets (objects of same area) is smaller for this case compared with the deterministic model. Otherwise, the quantity of $k$-sets is larger, it is done by $(l+1)(m+1)$.

The determination of the spectrum of fractal dimensions for the case of the multifractal is similar to the deterministic case, equation (5). The equation for $D_{k}$ in this case is the following:

$$
D_{k}=\lim _{n \rightarrow \infty} \frac{\log C_{i}^{l} s_{1}^{i} r_{1}^{l-i} C_{j}^{m} s_{2}^{j} r_{2}^{m-j}}{\log \left(s_{1}+r_{1}\right)^{n}},
$$

where the set of $k$ corresponds to any combination of $l$ and $m$ such that $n=l+m$. The generalization of this formula for several $\rho_{i}$ (for the case where each $\rho_{i}$ obeys $r_{i}+s_{i}=$ $\left.r_{1}+s_{1}\right)$ is performed by the introduction of new terms $C_{j}^{m_{i}} s_{2}^{j} r_{2}^{m_{i}-j}$ in the numerator.

Figure 3 shows the curve in crescent order of fractal dimensions $D_{k}$. In the $y$-axis is plotted $D_{k}$ and in the $x$-axis, $(k / n)^{\star}$, the respective $k$-set. We notice that the $k$-sets in the $x$-axis are shown in order of crescent $D_{k}$. The area of the $k$-sets is normalized between zero and one. In the figure there are three curves $\rho_{1}=1 / 4$ (point line), $\rho_{2}=2 / 3$ (dashed line), and the random curve (thick line). In this simulation we use $n=100$. The random curve is defined as the spectrum that results from the random choice between $\rho_{i}$ and $\rho_{2}$. The estimation of the random curve is performed assuming that for $n \rightarrow \infty$ we have $m=j=n / 2$.

A typical view of the the deterministic and the random multifractals is shown in figure 4. These two pictures show the deterministic multifractal (a) and one realization of the random multifractal (b). In figure 4 (a) we use $\rho=1 / 5$ and in figure 4 (b) $\rho_{1}=1 / 4$ and $\rho_{2}=2 / 3$. In both situations we evolved the algorithm until $n=10$.

\section{IV - FINAL REMARKS}

In this work we develop a random self-affine multifractal object that forms a tilling of the square. The multifractal is constructed by an algorithm that make successive sections of the square. In the special case where the section ratio is $1 / 2$ the algorithm generates the 
well known square lattice. At each $n$-step there is a random choice of partition parameter $\rho_{i}$. We explore in detail the case in which the ratio choice done is between two specific values $\rho_{1}$ and $\rho_{2}$. For this situation at the limit of infinite partition of the square, $n \rightarrow \infty$, we find analytically the full spectrum of fractal dimensions.

In the references [1, 2, 9] the deterministic multifractal object was used to study percolation properties and the breaking of universality class in two dimensions. In fact, the critical exponent related to the correlation length for the deterministic multifractal is not universal, it means, it is not the same as in standard percolation in two dimensions. This fact is related with the breaking of local isotropy. There are some points to consider in the symmetry properties of this multifractal object and its isotropy. The first is related with the stretching of the blocks of the multifractal which increases as $\rho \rightarrow 0$. Moreover the topology of multifractal, specially its coordination number, changes along the object. The object we develop in this work is a natural candidate to extend the cited papers to a non deterministic analyses of the breaking of universality class. In special we intend to analyze the reason for the breaking of universality class in the random multifractal and how the local isotropy affects this process.

The authors gratefully acknowledge the financial support of Conselho Nacional de Desenvolvimento Científico e Tecnológico (CNPq)-Brazil, FINEP and CTPETRO.

[1] G. Corso, J. E. Freitas, L. S. Lucena, R. F. Soares, accepted in Phy. Rev. E (2003), cond-mat/0212530

[2] L. S. Lucena, J. E. Freitas, G. Corso, and R. F. Soares, Brazilian Journal of Physics 33637 (2003).

[3] J. Muller, Annales Geophysicae - Atmospheres Hydrospheres and Space Sciences 11 (6): 525 (1993). 
[4] P. N. Khue, O. Fluseby, A. Saucier, J. Muller, Journal of Physics - Condensed Matter 14 (9): $2347(2002)$.

[5] R. H. Riedi, Multifractals and wavelets: a potential tool in Geophysics, Proceedings of the 68th SEG Meeting, New Orleans, Louisiana, USA, (1998).

[6] P. Hubert, Hydrological Sciences Journal 46897 (2001).

[7] S. Sachs, S. Lovejoy, D. Schertzer, Fractals 10253 (2002).

[8] M. Barnsley, Fractals Everywhere, (Academic Press, Boston, 1988).

[9] J. E. Freitas, G. Corso, and L. S. Lucena, cond-mat/0310779 (2003).

\section{FIGURE LEGENDS}

FIG. 1: The initial two steps in the formation of the deterministic multifractal. In (a) a vertical line cut the square in two pieces according to $\rho$. Two horizontal lines sectioning the rectangles by the same ratio are depicted in (b). The same process is repeated $n$-times inside each block. At the limit $n \rightarrow \infty$ we have a multifractal.

FIG. 2: The spectrum of fractal dimensions, $D_{k}$, for the deterministic multifractal. In the $y$-axis is $D_{k}$ and in the $x$-axis the respective normalized $k$-set. The curves for several $\rho$ are indicated in the figure.

FIG. 3: The spectrum $D_{k}$ for $\rho_{1}=1 / 4$ (point line) and $\rho_{2}=2 / 3$ (dashed line). In thick line is shown the random curve. In the the $x$-axis we show $(k / n)^{\star}$ the $k$-sets in order of crescent $D_{k}$. The area of the $k$-sets are normalized to one. 
FIG. 4: The random multifractal tilling evolved until $n=10$ for (a) the deterministic multifractal $(\rho=1 / 3)$ and $(\mathrm{b})$ one realization of the random multifractal (for $\rho_{1}=1 / 4$ and $\rho_{2}=2 / 3$ ). 


$$
\theta
$$




$$
\square
$$




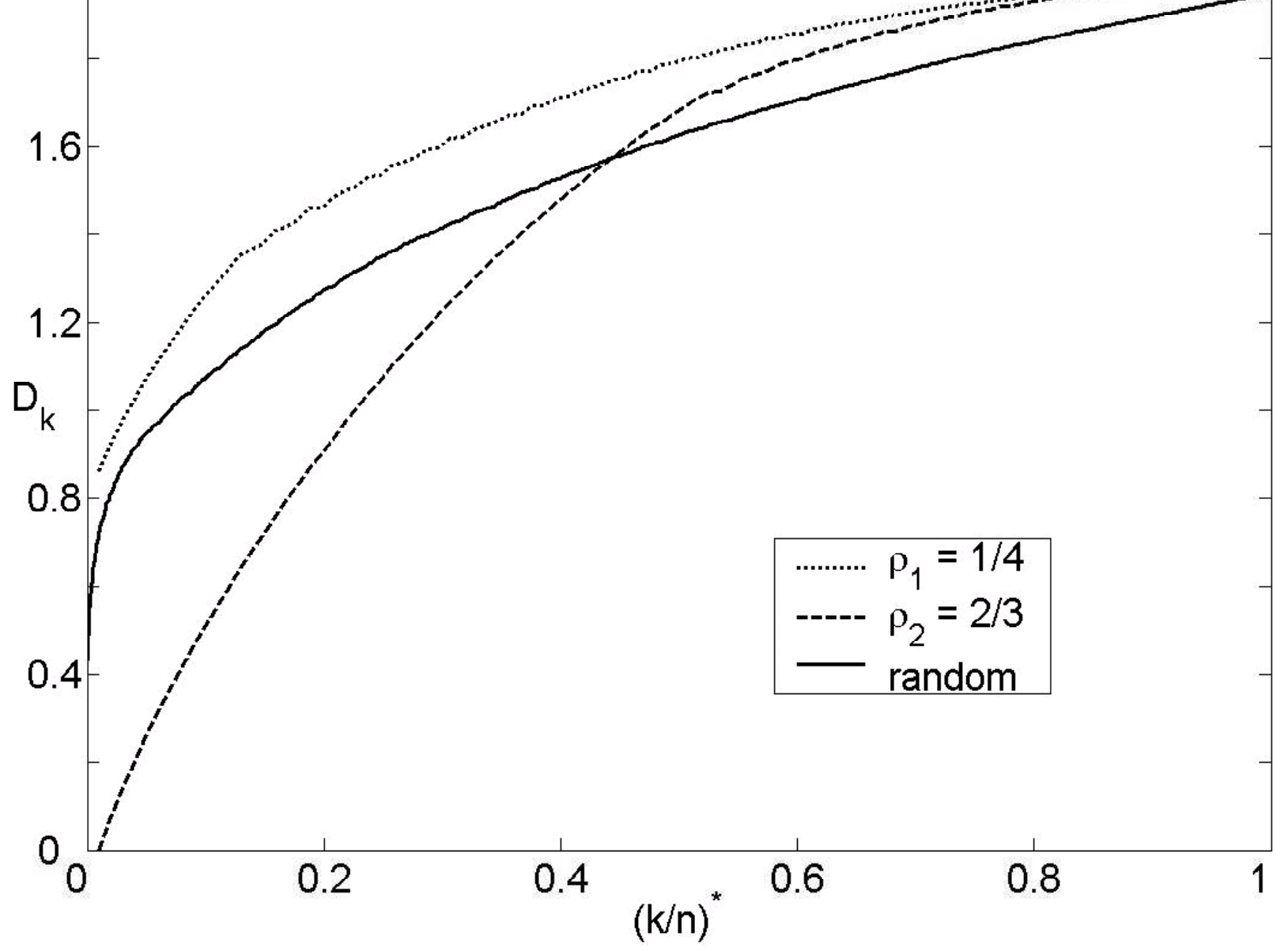




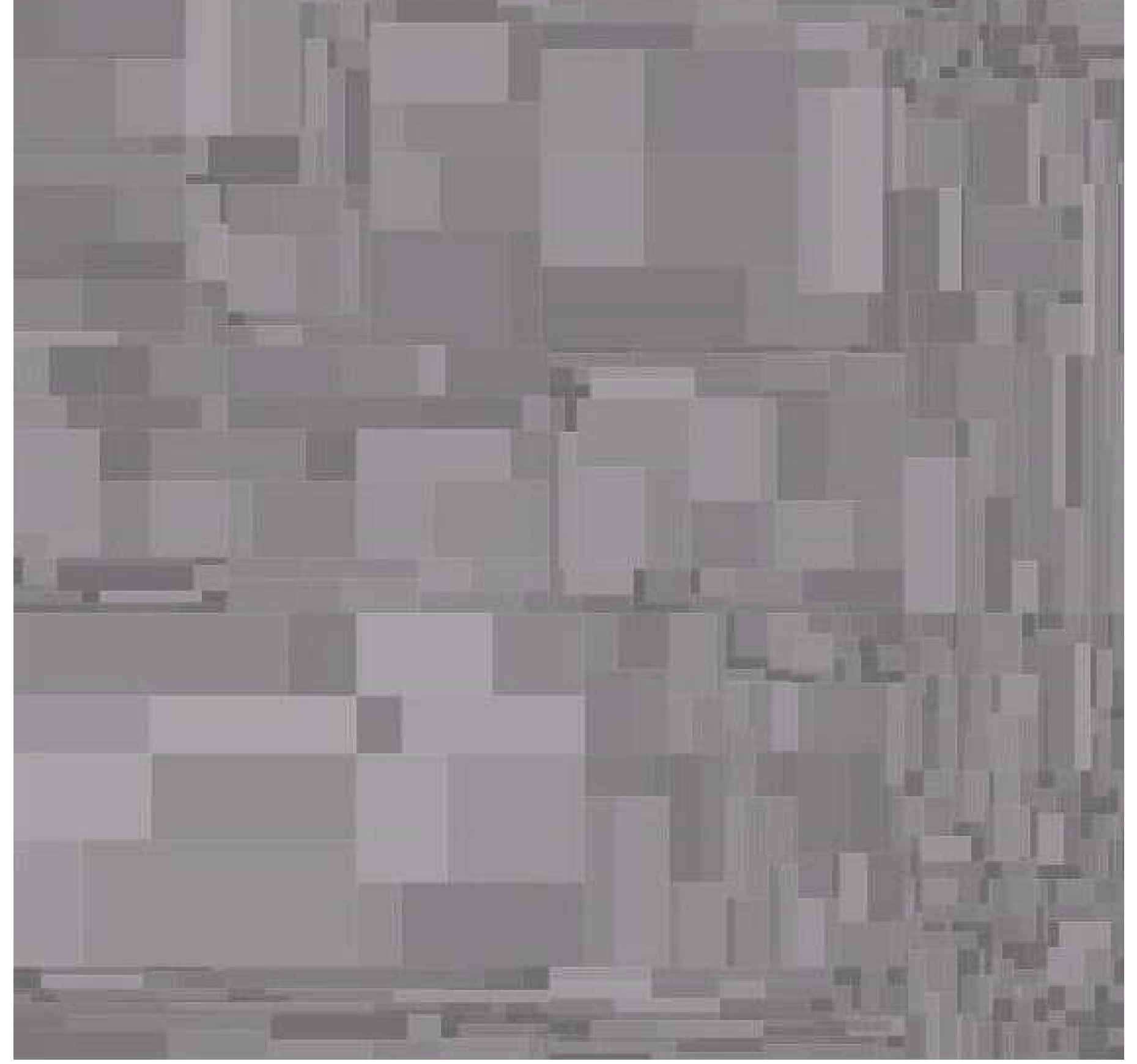

